\title{
ON QUASI-CONTINUOUS RINGS
}

\author{
W. K. NICHOLSON AND M. F. YOUSIF \\ (Communicated by Lance W. Small)
}

\begin{abstract}
A well-known result of Utumi asserts that a two-sided continuous two-sided artinian ring is quasi-Frobenius. In this paper we extend Utumi's result to quasi-continuous rings.
\end{abstract}

\section{INTRODUCTION AND DEFINITIONS}

A well-known result of Utumi [7] asserts that a two-sided continuous twosided artinian ring is quasi-Frobenius. In [4] this result was extended to twosided continuous rings with ACC on essential left and right ideals. In [3], motivated by a result of Carl Faith on self-injective rings, it was shown that a two-sided continuous ring with ACC on left annihilators is quasi-Frobenius. There are examples of two-sided artinian one-sided continuous rings which are not quasi-Frobenius (see [4]). In this paper we extend Utumi's result to quasicontinuous rings.

Throughout this paper all rings considered are associative with identity and all modules are unitary $R$-modules. We write $J(M), Z(M)$, $\operatorname{Soc}(M)$, and $E(M)$ for the Jacobson radical, the singular submodule, the socle, and the injective hull of ${ }_{R} M$, respectively. For any subset $X$ of $R, l_{R}(X)$ represents the left annihilator of $X$ in $R$.

Consider the following conditions on a module ${ }_{R} M$ :

(C1) Every submodule of $M$ is essential in a summand of $M$.

(C2) Every submodule isomorphic to a summand of $M$ is itself a summand.

(C3) If $M_{1}$ and $M_{2}$ are summands of $M$ with $M_{1} \cap M_{2}=0$, then $M_{1} \oplus M_{2}$ is a summand of $M$.

$M$ is called continuous if it satisfies conditions $(\mathrm{C} 1)$ and $(\mathrm{C} 2)$, quasi-continuous if it satisfies (C1) and (C3), and a CS-module if it satisfies condition (C1) only.

It is easy to see that $(\mathrm{C} 2)$ implies $(\mathrm{C} 3)$, but the converse is not true in general. Thus, every continuous module is quasi-continuous. The ring of integers $\mathbb{Z}$ is

Received by the editors July 23, 1992.

1991 Mathematics Subject Classification. Primary 16L60, 16P20, 16D50.

Key words and phrases. Continuous rings, quasi-continuous rings, self-injective rings, quasiFrobenius rings, chain conditions.

This research was supported by NSERC Grant A8075. The research of the second author was also supported by The Ohio State University in the form of a Special Research Assignment Quarter and a research grant. 
an example of a commutative, noetherian, quasi-continuous ring which is not continuous (and hence not quasi-Frobenius). For a full account of the subject of continuous and quasi-continuous modules we refer the reader to [5].

\section{THE RESULT}

Theorem 1. A left artinian two-sided quasi-continuous ring is quasi-Frobenius.

Before we begin the proof we need the following lemmas.

Lemma 1. $\bigoplus_{i=1}^{n} M_{i}$ is quasi-continuous if and only if each $M_{i}$ is quasicontinuous and $M_{j}$-injective for all $j \neq i$.

Proof. See [5, Corollary 2.14].

Lemma 2. Let $M_{1}$ and $M_{2}$ be summands of a quasi-continuous module $M$. If $E\left(M_{1}\right) \cong E\left(M_{2}\right)$, then $M_{1} \cong M_{2}$.

Proof. See [5, Theorem 2.31].

Lemma 3. Let $R$ be a left quasi-continuous ring. Then $R$ is left continuous if and only if $Z\left({ }_{R} R\right)=J(R)$ and $R / J(R)$ is regular.

Proof. See [5, Proposition 3.15].

Lemma 4. Let $R$ be a two-sided continuous ring with ACC on left annihilators. Then $R$ is a quasi-Frobenius ring.

Proof. See [3, Theorem 1].

Lemma 5. Let $R$ be a semiperfect ring. Then $Z\left({ }_{R} R\right) \subseteq J(R)$ and $Z\left(R_{R}\right) \subseteq$ $J(R)$.

Proof. Since $R$ is semiperfect, $R=R e \oplus R(1-e)$ such that $R e \subseteq Z\left({ }_{R} R\right)$, $Z\left({ }_{R} R\right) \cap R(1-e)$ is small in $R$, and $e^{2}=e \in R$. Since $Z\left({ }_{R} R\right)$ does not contain nonzero idempotents, $Z\left({ }_{R} R\right)$ is small in $R$. Thus $Z\left({ }_{R} R\right) \subseteq J(R)$. Similarly $Z\left(R_{R}\right) \subseteq J(R)$.

The next lemma is a key result for the proof of Theorem 1.

Lemma 6. Let $R$ be a semiprimary left quasi-continuous ring. Then $R$ is left continuous.

Proof. Since $R$ is semiperfect, we can write $R=\bigoplus_{i=1}^{n} R e_{i}$ a direct sum of principal indecomposable left ideals $R e_{i}$. Each $e_{i}$ is a nonzero primitive idempotent. By Lemma 1 , each $R e_{i}$ is quasi-continuous. Since an indecomposable $C S$-module is necessarily uniform, each $R e_{i}$ is uniform. Since $R$ is semiprimary, $\operatorname{Soc}\left(R e_{i}\right)$ is essential in $R e_{i}$, and hence each $R e_{i}$ has a unique minimal left ideal.

Without loss of generality we may assume that $\mathscr{E}=\left\{e_{1}, \ldots, e_{m}\right\}$ is a basic set of primitive idempotents for $R$. Let $T_{i}=\operatorname{Soc}\left(R e_{i}\right), 1 \leq i \leq m$. Hence $E\left(T_{i}\right)=E\left(R e_{i}\right)$.

Claim. $T_{i} J e_{k}=0$, for every $i$ and $k, 1 \leq i, k \leq m$.

Suppose $k \neq i$ and $T_{i} a e_{k} \neq 0$ for some $a \in J$. It is easy to see that $T_{i} \cong$ $T_{i} a e_{k}$ as minimal left ideals. Since $T_{i} a e_{k} \subseteq R e_{k}$, we infer that $T_{k}=T_{i} a e_{k}$. Since $T_{i} \cong T_{k}, E\left(R e_{i}\right) \cong E\left(R e_{k}\right)$. Now an application of Lemma 2 ensures that $R e_{i} \cong R e_{k}$, and hence $i=k$, a contradiction. Now, if $k=i$ and $T_{i} a e_{i} \neq 0$ for some $a \in J$, then $T_{i} a e_{i} \cong T_{i}$, and hence $T_{i}=T_{i} a e_{i}$ whence $T_{i} J e_{i}=T_{i}$. 
Let $e=e_{1}+\cdots+e_{m}$ be the basic idempotent. Then $T_{i} J e=T_{i} J e_{i}=T_{i}$. Thus $T_{i} J=T_{i} R$. Since $R$ is semiprimary, $J^{n-1} \neq 0$ and $J^{n}=0$ for some $n \geq 1$. Thus $\left(T_{i} J\right)\left(J^{n-1}\right)=\left(T_{i} R\right)\left(J^{n-1}\right)$, and hence $T_{i} J^{n-1}=0$. By repeating the argument, if necessary, we get $T_{i} J=0$. Thus $T_{i} R=0$, and hence $T_{i}=0$, a contradiction. Thus $T_{i} J e_{k}=0$ in every case, proving the claim.

Since $T_{i} J e_{k}=0$ and $T_{i} J e=0$, we infer that $T_{i} \subseteq l_{R}(J), 1 \leq i \leq m$. Since $R / J$ is semisimple, $l_{R}(J)=\operatorname{Soc}\left(R_{R}\right)$, and hence $\operatorname{Soc}\left({ }_{R} R\right) \subseteq \operatorname{Soc}\left(R_{R}\right)$. Now since $\operatorname{Soc}_{R} R$ is essential in ${ }_{R} R$ and $\operatorname{Soc}\left({ }_{R} R\right) \subseteq l(J)$, it follows that $J(R) \subseteq Z\left({ }_{R} R\right)$. By Lemma 5 , we get $J(R)=Z\left({ }_{R} R\right)$. And, by Lemma $3, R$ is left continuous.

Now the proof of Theorem 1 is an easy consequence of Lemma 6 .

Proof of Theorem 1. By Lemma 6, $R$ is a two-sided continuous ring, and, by Lemma $4, R$ is a quasi-Frobenius ring.

Corollary 1. Let $R$ be a left quasi-continuous ring with DCC on essential left ideals. Then $R$ is a left continuous ring.

Proof. By [1, Proposition 1.1], $R / \operatorname{Soc}\left({ }_{R} R\right)$ is left artinian. And, by [2, Corollary 6], $R$ is left artinian. Then it follows from Lemma 6 that $R$ is left continuous.

Corollary 2. Let $R$ be a two-sided quasi-continuous ring with DCC on essential left ideals. Then $R$ is quasi-Frobenius.

Remark. There is an example of a commutative, local, semiprimary, continuous ring which is not injective (see Rizvi [6]).

\section{REFERENCES}

1. E. P. Armendariz, Rings with dcc on essential left ideals, Comm. Algebra 8 (1980), 299-308.

2. V. Camillo and M. F. Yousif, CS-Modules with acc or dcc on essential submodules, Comm. Algebra 19 (1991), 655-662.

3. __ Continuous rings with ACC on annihilators, Canad. Math. Bull. 34 (1991), 462-464.

4. S. K. Jain, S. R. López-Permouth, and S. T. Rizvi, Continuous rings with acc on essentials are artinian, Proc. Amer. Math. Soc. 108 (1990), 583-586.

5. S. H. Mohamed and B. J. Müller, Continuous and discrete modules, London Math. Soc. Lecture Notes Ser., vol. 147, Cambridge Univ. Press, Cambridge and New York, 1990.

6. S. T. Rizvi, Commutative rings for which every continuous module is quasi-injective, Arch. Math. (Basel) 50 (1988), 435-442.

7. Y. Utumi, On continuous rings and self-injective rings, Trans. Amer. Math. Soc. 118 (1965), 158-173.

Department of Mathematics and Statistics, The University of Calgary, 2500 University Drive N.W., Calgary, Alberta, Canada T2N 1N4

E-mail address: wknichollacs.ucalgary.ca

Department of Mathematics, Ohio State University, Lima, Ohio 45804

E-mail address: yousif. 1 cosu.edu 